\title{
Using lasers for cleaning ceramic and plaster patrimonial objects.
}

\section{Nathalie HUET $^{1 *}$, Eric TANGUY ${ }^{2}$, Armand VINÇOTTE ${ }^{1}$, Vassilis ZAFIROPULOS ${ }^{3}$}

\author{
'Arc'Antique - 26, rue de la Haute Forêt - 44300 NANTES (France) - arc.antique@ wanadoo.fr \\ ${ }^{2}$ LPIO - Faculté des Sciences et des Techniques de Nantes - 2, rue de la Houssinière - 44000 NANTES \\ (France) - eric.tanguy@ @hysique.univ-nantes.fr \\ ${ }^{3}$ Laser and Applications Division, IESL - FORTH - Vasilika Vouton - P.O. Box 1527 - HERAKLION \\ 71110 - Crete (Greece) - zafir@iesl.forth.gr
}

\begin{abstract}
Traditional cleaning techniques (sandblasting, chemical, ...) turn out to be ineffective or damaging on some ceramics and plaster objects. It's especially true on outdoor ceramics covered with black crust or indoor biscuits wares and plasters exposed to dust and repeated handling. The use of lasers to remove dirt, such as for stone, has then been considered. This paper study the behaviour of these materials under the impact of different type of lasers irradiation (Nd:YAG first and third harmonic, excimer, ...). According to the type of dirt and underlying material, the effectiveness of cleaning depends on the laser wavelength and its energy density. The results are satisfactory with no damage and no coloration change on plaster cleaning by Nd:YAG third harmonic.
\end{abstract}

\section{INTRODUCTION}

The use of Nd:YAG lasers to remove dirt from stone monuments is gradually replacing [i] the more abrasive technique of sandblasting. This trend has caused Arc'Antique to consider lasers as a solution for the cleaning of outdoor glazed ceramics (stoneware bowls) covered with a blackish layer of pollution (Figure 1). In fact, the standard techniques using chemical products have proved totally ineffective on these objects and sandblasting is relatively damaging to the glaze. The promising results obtained when cleaning glazed ceramics [ii] suggested to us that the technique could be also used for some indoor ceramics, like biscuit ware, and plasters. These materials are indeed often covered in museum dirt (dust, grease, etc.) that is difficult to remove without damaging the object.

\section{THE CHOICE OF THE WAVELENGTH}

Following initial tests with an infrared Nd:YAG laser, it was decided that research have to be undertaken into the behaviour of ceramic and plaster materials under the impact of lasers, into the types of laser most suited to the removal of the most commonly found dirt, and into optimisation of the different operational parameters. The first study started in FORTH (Crete), where we were able to take advantage of the expertise of the ARTT team in the cleaning field and to use their high performance equipment (different lasers, on-site monitoring techniques). It consisted of identifying the materials cleaned and layers of dirt to be removed. We had the opportunity to try out the available cleaning options for different categories of dirt (pollution, paint, dust, oxide staining, grease) on several types of material (pottery, porcelain, biscuit ware, plaster) under the impact of different types of laser (Nd:YAG first and third harmonic, excimer). It was demonstrated that the effectiveness of this cleaning is complex, as, for each type of dirt and underlying material, the behaviour differs depending on the type of laser used (and therefore the wavelength of the laser) due to the physical mechanism of ablation involved (photothermal/photochemical). Some results appeared really encouraging, such as the removal, with an excimer laser $(\mathrm{KrF}, \lambda=248 \mathrm{~nm})$, of layers of dust from "rough" porous and fragile objects, such as biscuit or plaster, with no damage. Similarly, spectacular results can be obtained with a third harmonic Nd:YAG $(\lambda=355 \mathrm{~nm})$ in removing accumulated dirt caused by external pollution from certain glazed ceramics (Figure 2) or dust on rough clay mass and plaster. Highly enlarged images demonstrate that the surface is not damaged, unlike with standard techniques such as micro sandblasting.

\section{PROBLEMS AND CAUSES}

Despite these good results, certain tests were catastrophic. A number of observations are leading us to make a more detailed study of phenomena using SEM, EDS, X-Ray diffraction, infrared spectrometry. The first problem is due to the thermal effect during the use of $\mathrm{Nd}$ :YAG lasers $(\lambda=1.06 \mu \mathrm{m})$ provoking cracking and fusion of glazes that can be avoided with a precise control of the fluence. But the major effect is a change of coloration which affect glazes, painting, clay mass and plaster. Looking closely at these disappointing tests, we tried to analyse the causes or at less the material on wich these phenomena appeared. Two problems were point out on certain materials depending of the wavelenghts used : a yellowing [iii] and colour alterations. With the infrared Nd:YAG laser, usually used for stone, the surface becomes yellowish (Figure 2) or greyish. If on stone it could be identified as patina, the same explanation is not available on ceramics where ageing cause no yellowing. It seems that this colour is not caused by an alteration of the material but rather a layer of material (dirt?) redeposited or still deposited on the surface. Although, as many others researchers, it has not yet been possible to identify this layer even using sophisticated techniques (ESCA). Nevertheless, a second cleaning with another type of laser, such as the third harmonic Nd:YAG, can totally remove it and restore the 
original colour of the object. It is particularly observable on plaster : during cleaning with an infrared Nd:YAG laser an intense yellowing appeared (Figure 3a) removable by using after a third harmonic Nd:YAG (Figure 3c) with no apparent damage on the surface, but perhaps a too-whitening surface comparing to traditional cleaning methods. Furthermore, on a certain number of materials we observed more or less lasting colour modifications generally involving a greying or blackening. Two cases can be distinguished. Some glazes cleaned by excimer laser develop sometimes temporary iridescences on the surface, which might be explained by a photochemical change in the glass (ultraviolet creating molecular disorder and a change in colour centres). Other materials (paints, sediment, concretions, etc.), which contain metallic oxydes, also change colour under the action of the laser [iv] (Figure 4). The explanation could be that the metal elements undergo a valence state change (oxidation or reduction) causing this colour change. To complete these observations, others tests are being conducted on synthetic samples better suited to more sophisticated analyses (ESCA, X-ray grazing incidence diffraction, Mössbauer, etc.).

\section{CONCLUSIONS}

An exhaustive study based on the most common wavelengths should validate these hypotheses and refine the efficacy of these lasers depending on the materials and dirt. The complementarity of certain types of wavelengths have to be studied in detail, such as for example using an Nd: YAG laser at $1.06 \mu \mathrm{m}$ to remove thick layers of pollution followed by an Nd: YAG third harmonic to eliminate the residual yellowish layer. In some cases, as fragile objects, using lasers for the cleaning is the only potential technic against traditionnal methods to avoid surface damages (pits, abrasion, remains of chemical products...).

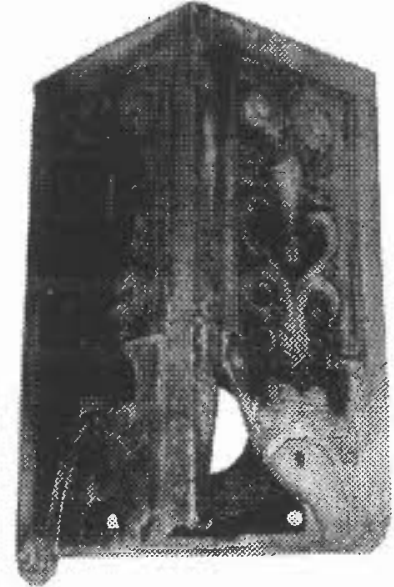

Figure 1 : Example of outdoor ceramic covered with black crust

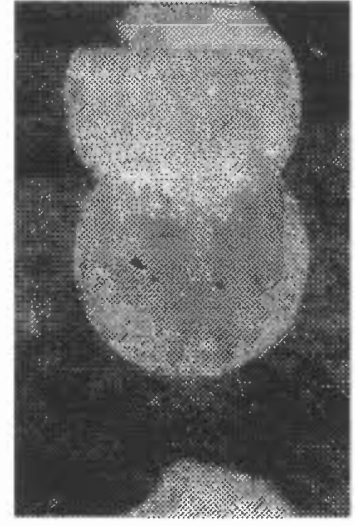

Figure 2 : Ceramic cleaned by Nd:YAG first harmonic (middle circle) and third harmonic (bottom circle)

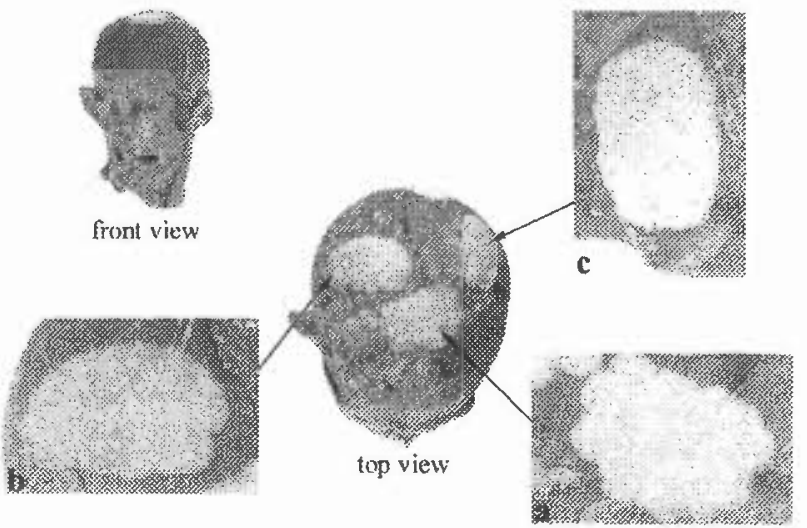

Figure 3 : Plaster écorché cleaned by Nd :YAG

a) first harmonic

b) second harmonic

c) third harmonic

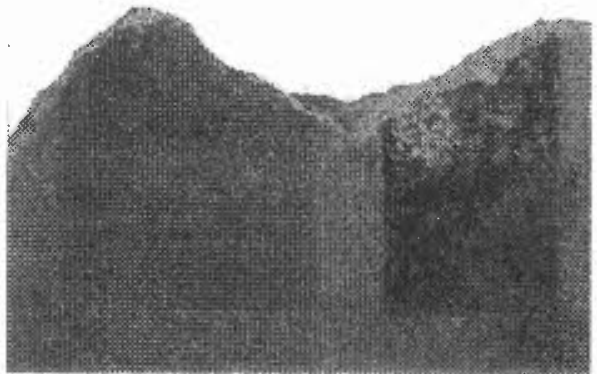

Figure 4 : Changed of coloration on a rough clay ceramic

Acknowledgements: Arc'Antique analysed the results of the first tests conducted in 1997 in FORTH (Crete) with different types of lasers through a program financed by the LUF (Laser Ultraviolet Facility). This program has benefited from multi-year financial assistance of C2RMF (Centre de Restauration et de Recherche des Musées de France).

\section{REFERENCES}

i. M. Cooper, Laser cleaning in conservation, an introduction (Butterworth Heinmann, Oxford, 1998).

ii. P. Gaspar et al., Studies in conservation, 45, 189-200 (2000).

iii. V. Zafiropoulos et al., Proceedings of Lacona IV, Paris, 2001.

iv. M. Chappé, J Hildenhagen, K. Dickmann, Proceedings of Lacona IV, Paris, 2001. 